\title{
Computation of quantum bound states on a singly punctured two-torus
}

\begin{abstract}
We study a quantum mechanical system on a singly punctured two-torus with bound states described by the Maass waveforms which are eigenfunctions of the hyperbolic LaplaceBeltrami operator. Since the discrete eigenvalues of the Maass cusp form are not known analytically, they are solved numerically using an adapted algorithm of Hejhal and Then to compute Maass cusp forms on the punctured two-torus. We report on the computational results of the lower lying eigenvalues for the punctured two-torus and find that they are doubly-degenerate. We also visualize the eigenstates of selected eigenvalues using GridMathematica.
\end{abstract}

Keyword: Algebraic methods; Quantum mechanical system; Singly punctured two-torus. 